\title{
Modal Analysis of Nanoparticles Reinforced Natural Rubber
}

\author{
Ramzan Muhammad ${ }^{1}$, Bimlesh Kumar ${ }^{2}$, Atul Chaskar $^{3}$ \\ ${ }^{1}$ MMANTC, Malegaon; 3158, Lane No.1, Akbar Chawk, Dhule-424001 (M.S.) India \\ ${ }^{2}$ Department of Mechanical Engineering, J T Mahajan College of Engineering, Faizpur, Dist. Jalgaon \\ ${ }^{3}$ Department of Dyestuff Technology, Institute of Chemical Technology, Matunga, Mumbai
}

\begin{abstract}
The voids in microstructural network of rubber favors the reinforcement of micro and nanosized fillers with a wide range of physical and mechanical properties. This phenomenon of nanofiller reinforcement opens a new horizon for researchers with a tremendous scope to design and investigate nanocomposite materials especially for energy storage, shock absorbing and vibration damping applications. In this study the possibilities of designing a new nanocomposite vibration damper is investigated through the mode of reinforcement of alumina-zirconia $\left(\mathrm{Al}_{2} \mathrm{O}_{3}-\mathrm{ZrO}_{2}\right)$ hybrid nanocomposite particles in natural rubber (NR). TEM images of nanoparticles indicate clearly the particle size and reinforcement of $\mathrm{ZrO}_{2}$ in $\mathrm{Al}_{2} \mathrm{O}_{3}$ matrix and SEM images of nanocomposite NR shows the surface morphology and entanglement of elastomeric chains. Finite element method is applied to validate the results and it was recorded that the free vibration amplitude decay time is reduced by $25 \%$ alongwith excellent $260 \%$ enhancement in storage modulus with $15 \%$ filler concentration. A sensitivity analysis is carried out for the improvement in storage modulus of nanocomposite rubber as a function of nanofiller concentration.
\end{abstract}

Keywords: Finite Element Analysis (FEA), Loss Factor, Nano composites, Transmission Electron Microscopy (TEM), Vibration Damping

\section{Introduction}

Rubber, technically elastomer, is a unique material which is both elastic and viscous [1] and consists of innumerable microsized spring-mass dashpot systems. The spring justifies the elasticity which obeys Hook's law and increases with increase in stress whereas the dashpot exhibits viscosity which follows the Newton's law of viscosity and increases with the strain rate. Few of these elastomers strain crystallize and the others require reinforcement to obtain the adequate static and dynamic characteristics. Natural Rubber (NR) and graphene nanocomposites increase the electrical conductivity and cause an important enhancement on the mechanical behavior of the NR [2]. Styrene-butadiene rubber/clay nanocomposite can enhance the modulus eight times at $300 \%$ strain and tensile strength seven times compared with cured pure rubber [3]. Active nanoparticles can form physical or chemical bonding with rubber matrix and enhance exceptionally the mechanical properties of the nanocomposites. The chemical bonding may develop a solid solution like strengthening mechanism by creating a solute-solvent relationship between the reinforced nanoparticles and rubber base $[4,5]$. Natural rubbers combine high tensile strength, flexibility, and tear strength with outstanding resistance to fatigue $[6,7]$. Alumina $\left(\mathrm{Al}_{2} \mathrm{O}_{3}\right)$ is a potential candidate as the dispersed phase in the natural rubber matrix $[8,9]$. Factors such as size distribution, shape, volume fraction, permittivity, and conductivity of the particles and the host solvent, have been well known to affect the electrical and rheological properties of the polyisoprene based blends or composites [10-17]. For the products made of filled rubber compounds that operate under dynamic loads, such as the tire and air-spring [18] we still do not have a complete understanding of its mechanical behavior.

In this study, five samples of natural rubber reinforced with different concentration of Alumina $\left(\mathrm{Al}_{2} \mathrm{O}_{3}\right)$-Zirconia $\left(\mathrm{ZrO}_{2}\right)$ hybrid nanocomposite particles are examined and compared for some dynamic mechanical properties by using analytical method and finite element method. Nanocomposite sample is modeled as simply supported beam subjected to initial excitation and eigenvalues, eigenvectors and lateral displacement relationship are derived. A sensitivity analysis [19] for storage modulus as a function of nanofiller concentration is carried out, which provides an insight to establish a relationship of dynamic property variation in nanocomposites.

\section{Experimentation And Sample Preparation}

Alumina-zirconia $\left(\mathrm{Al}_{2} \mathrm{O}_{3}-\mathrm{ZrO}_{2}\right)$ nanocomposite particles were synthesized through sol-gel method with a minor modification in the protocol as reported by Sarkar et al. [20]. Instead of wet milling acetone washing was carried out to deagglomerate the nanoparticles after calcination. TEM images of nanoparticles and SEM images of nanocomposite NR are illustrated in the Figs. 1 and 2 respectively. The natural rubber (polyisoprene) as per the specifications of Rubber Research Institute of India (RRII), Kottayam (India) (ISNR-3L) with highest purity (dust content $<0.04 \%$ ) was obtained. ISNR-3L was a dried rubber in a light color sheet with specific 
gravity 0.93, storage modulus $1.56 \mathrm{MPa}$ and Mooney viscosity $63 \mathrm{MU}$. The compounding ingredients mentioned in the Table 1 are mixed in two roll mill to form nanocomposite rubber samples in the form of square cross-sectional strips of dimension ( $\mathrm{L}: \mathrm{W}: \mathrm{D}=150: 150: 2) \mathrm{mm}$ with $\mathrm{Al}_{2} \mathrm{O}_{3}-\mathrm{ZrO}_{2}$ nanocomposite particle concentrations varying from $5-15 \%(5,8,10,12,15 \%)$.

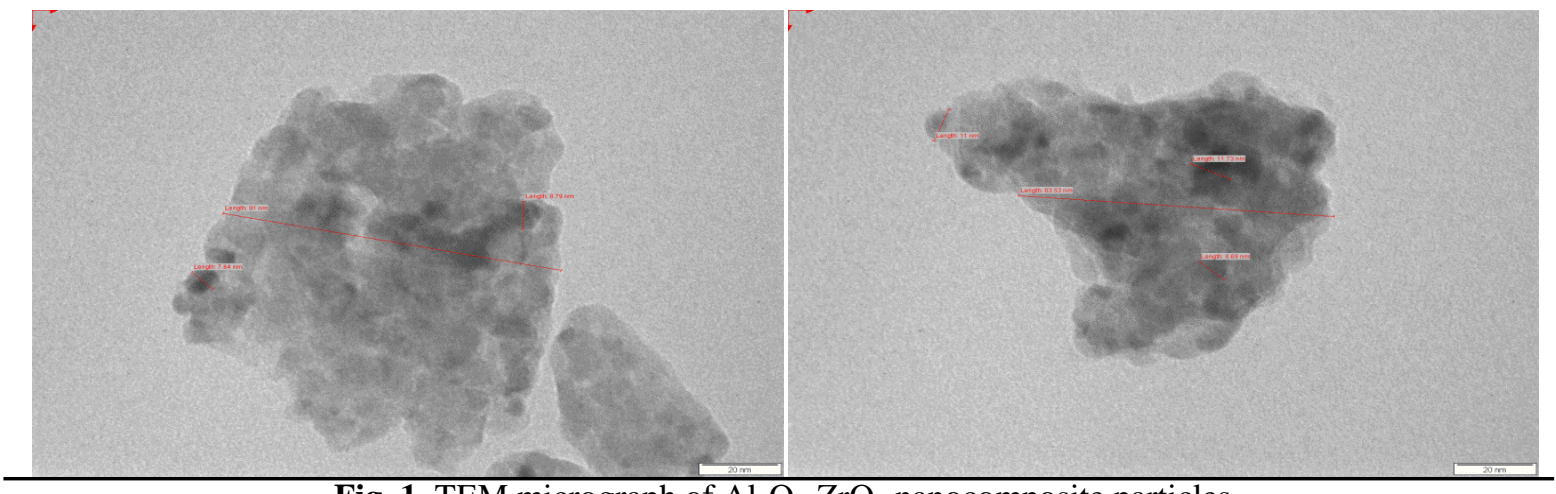

Fig. 1. TEM micrograph of $\mathrm{Al}_{2} \mathrm{O}_{3}-\mathrm{ZrO}_{2}$ nanocomposite particles

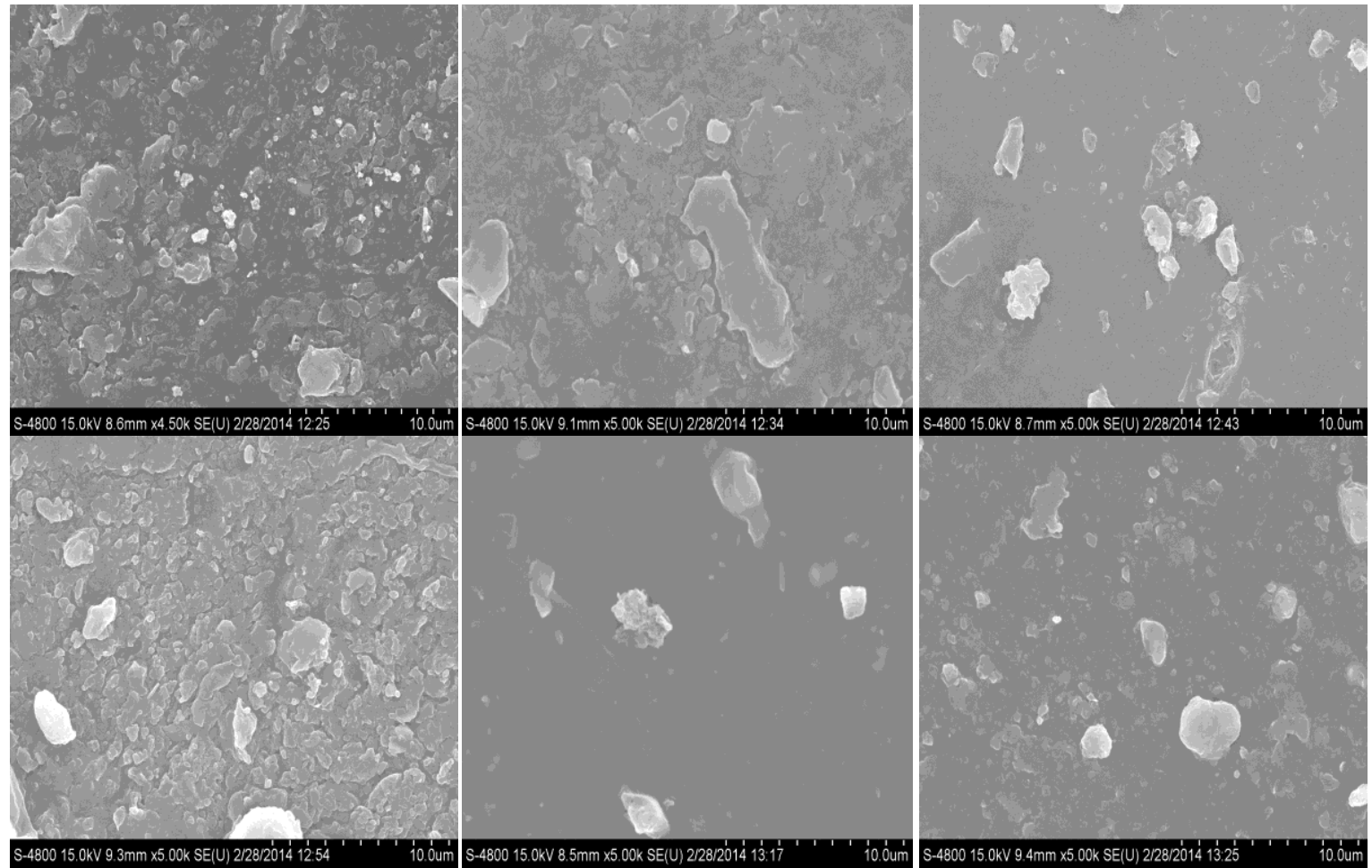

Fig. 2. SEM micrograph of nanocomposite NR with 0, 5, 8, 10, 12 and $15 \%$ nanofiller concentration respectively

Table1. Compounding ingredients of Natural Rubber-Nanofiller nanocomposite

\begin{tabular}{cllc}
\hline Sr. & Ingredients of Nanocomposite Natural & Function & Quantity (PHR) \\
No. & Rubber & Matrix & 100 \\
\hline 1 & Natural Rubber (NR) & Cross-linking and curing agent & 1.0 \\
2 & Sulpher & Accelerator & 1.5 \\
3 & MBT & Curing agent, accelerator, Anticoagulant & 5.0 \\
4 & Zinc Oxide $(\mathrm{ZnO})$ & Curing agent, accelerator, Anticoagulant & 2.0 \\
5 & $\mathrm{Stearic}$ acid & Nanofillers & $0-15$ \\
6 & $\mathrm{Al}_{2} \mathrm{O}_{3}-\mathrm{ZrO}_{2}$ Nanocomposite & & \\
\hline
\end{tabular}

MBT- Mercapto Benzothiazole

The initial properties of nanocomposite rubber such as density and storage modulus as a function of nanofiller concentration were evaluated using Halpin-Tsai approach [21] and recorded in the Table 2. 
Table2. Mechanical properties of Nanoparticles reinforced Natural Rubber (NR)

\begin{tabular}{|c|c|c|c|c|c|c|c|c|}
\hline \multirow{2}{*}{$\begin{array}{l}\text { Sr. } \\
\text { No. }\end{array}$} & \multirow{2}{*}{ Properties and notations used } & \multirow{2}{*}{ Unit } & \multicolumn{6}{|c|}{ Nanoparticles reinforced Natural Rubber (NR) } \\
\hline & & & $\mathbf{0 \%}$ & $5 \%$ & $8 \%$ & $10 \%$ & $12 \%$ & $15 \%$ \\
\hline 1 & Density $(\rho)$ & $\mathrm{Kg} / \mathrm{m}^{3}$ & 930 & 1025 & 1095 & 1145 & 1198 & 1281 \\
\hline 2 & $\begin{array}{l}\text { Elastic Modulus (E) (500\% } \\
\text { Elongation) }\end{array}$ & MPa & 1.56 & 1.98 & 2.40 & 2.78 & 3.23 & 4.05 \\
\hline
\end{tabular}

This model assumes an isotropic particulate reinforcement of the matrix. The following relations were used to evaluate the property;

where;

$$
\begin{gathered}
P=P_{m} \frac{\left(1+\varphi e_{P} v_{f}\right)}{\left(1-e v_{f}\right)} \\
e_{P}=\frac{\left(P_{f}-P_{m}\right)}{\left(P_{f}+\varphi P_{m}\right)} \\
\varphi=\left[2+\left(40 * v_{f}\right)\right]
\end{gathered}
$$

In the above Eqs. (1-3), $\mathrm{P}$ stands for a property such as density, modulus of elasticity etc. and suffixes $\mathrm{m}$ and $\mathrm{f}$ corresponds to matrix and filler respectively. The term $\mathrm{e}_{\mathrm{P}}$ is a factor corresponding to a property which depends on another factor $\varphi$ also varying with volume fraction $\left(\mathrm{v}_{\mathrm{f}}\right)$ of the filler.

\section{Modeling}

The nanocomposite sample was modeled as Euler-Bernoulli beam as shown in Fig. 3 for analytical calculations and the equation of motion is obtained as below; [22]

$$
\text { EI } \frac{\partial^{4} \mathrm{y}}{\partial \mathrm{x}^{4}}+\rho \mathrm{A} \frac{\partial^{2} \mathrm{y}}{\partial \mathrm{t}^{2}}=0
$$

Besides the assumptions of homogeneity and uniformity of nanoparticles size and distribution the above equation of motion, Eq. (4) was derived considering the following assumptions:

i) No axial force is acting on the beam.

ii) Warping, shear deformation and Poisson effect are negligible

iii) Bending and twisting moments at any arbitrary position along the beam are proportional [23].

Considering nanocomposite (visco-elastic) beam subjected to initial excitation, which is a case of beam under damped-free vibration, the modified equation of motion from Eq. (4) can be obtained as;

The solution of the Eq. (5) can be obtained as;

$$
\text { EI } \frac{\partial^{4} \mathrm{y}}{\partial \mathrm{x}^{4}}+\rho \mathrm{A} \frac{\partial^{2} \mathrm{y}}{\partial \mathrm{t}^{2}}+\mathrm{c} \frac{\partial \mathrm{y}}{\partial \mathrm{t}}=0
$$

$$
y(x, t)=A \cosh \beta x+B \sinh \beta x+C \cos \beta x+D \sin \beta x
$$

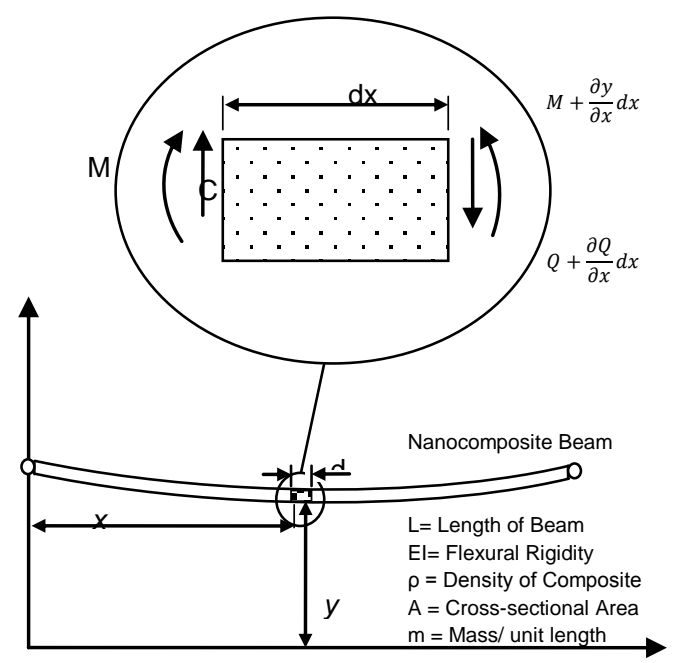

Fig. 3. Model of nanocomposite as simply supported beam

Applying the boundary conditions of simply supported beam to Eq. (6);

$$
\text { At } \mathrm{x}=0 ; \quad \mathrm{y}(0, \mathrm{t})=0 \quad\left[\frac{\partial^{2} \mathrm{y}}{\partial \mathrm{t}^{2}}\right]_{\mathrm{x}=0}=0
$$




$$
\text { At } \mathrm{x}=\mathrm{L} ; \quad \mathrm{y}(\mathrm{L}, \mathrm{t})=0 \quad\left[\frac{\partial^{2} \mathrm{y}}{\partial \mathrm{t}^{2}}\right]_{\mathrm{x}=\mathrm{L}}=0
$$

Constants $\mathrm{A}, \mathrm{B}$ and $\mathrm{C}$ become zero and the form of solution can be expressed as;

$$
\mathrm{y}(\mathrm{x}, \mathrm{t})=\sum_{\mathrm{n}=1}^{\infty} \mathrm{D}_{\mathrm{n}} \sin \left(\frac{\mathrm{n} \pi}{\mathrm{L}}\right) \mathrm{x}
$$

The relation between the eigenvalues and undamped natural frequencies is given by;

$$
\omega_{\mathrm{n}}=\beta^{2} \sqrt{\frac{\mathrm{EI}}{\rho \mathrm{A}}}
$$

where, the eigenfunction $\beta=n \pi / L$; the corresponding natural frequencies can be obtained by Eq. (8), $(\mathrm{n}=1$ to $\infty$ ) and the respective mode shapes can be determined by using Eq. (7). The Eq. (5) can be expressed as a set of ordinary differential equations in the modal coordinates;

where,

$$
\ddot{y}_{1}+2 \omega_{i} \xi_{i} \dot{y}_{i}+\omega_{i}^{2} y_{i}=0
$$

$$
\xi_{\mathrm{i}}=\frac{\mathrm{c}}{2 \mathrm{~m} \omega_{\mathrm{i}}} \quad(\mathrm{i}=1,2, \ldots)
$$

The damping ratio $(\xi)$ is a function of viscous damping constant (c) and frequency $(\omega)$. The solution of the above Eq. (9) can be extracted as;

$$
y(x, t)=\left[e^{-\zeta \omega_{i} t}\left(\cos \omega_{d i} t+\frac{\zeta_{i} \sin \omega_{d i} t}{\sqrt{1-\zeta_{i}^{2}}}\right)\right]
$$

where

$$
\omega_{d i}=\omega_{i} \sqrt{1-\zeta_{i}^{2}}
$$

The above response Eq. (11) was simulated in MATLAB and the output for initial excitation corresponding to the fundamental mode of vibration was plotted.

\section{Analysis By Finite Element Method}

The free body diagram of finite elements with nodal points for a simply supported beam is illustrated in the Fig. 4. Assume the discretization of uniform beam with 10 finite elements to represent the bending vibration in terms of two displacements at each nodal point of the element as shown in the enlarged view of a single element in Fig. 4.

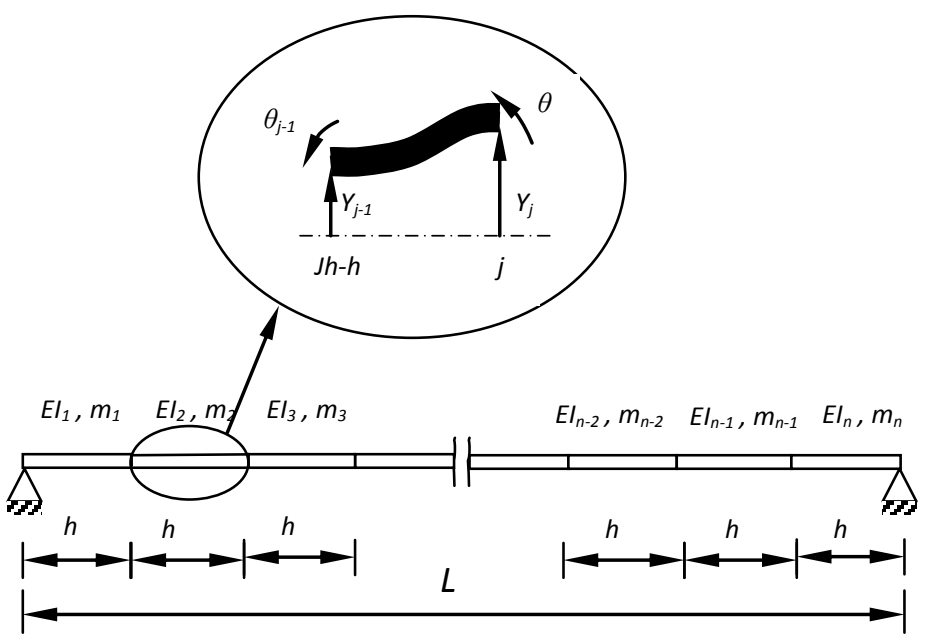

Fig. 4. Model of discretized simply supported nanocomposite beam; Two displacements at each nodal point is shown in the enlarged view of an element Eq. (13) [22];

The characteristic equation for eigenvalue problem in bending vibration of a beam by FEM is given by

$$
K a=\lambda M a
$$

where $\mathrm{K}$ and $\mathrm{M}$ are global stiffness and mass matrices respectively, a is system nodal vector and $\lambda$ is eigenvalue and expressed as;

$$
\lambda=\omega^{2}
$$

The element stiffness matrices for a constant value of $\mathrm{EI}_{\mathrm{j}}$ can be obtained as; 


$$
K_{j}=\frac{E I_{j} n^{3}}{L^{3}}\left[\begin{array}{cccc}
12 & 6 & -12 & 6 \\
6 & 4 & -6 & 2 \\
-12 & -6 & 12 & -6 \\
6 & 2 & -6 & 4
\end{array}\right] \quad \mathrm{j}=1,2, \ldots, \mathrm{n}
$$

The element mass matrices for a constant value of $\mathrm{m}_{\mathrm{j}}$ can be obtained as;

$$
M_{j}=\frac{m_{j} L}{420 n}\left[\begin{array}{cccc}
156 & 22 & 54 & -13 \\
22 & 4 & 13 & -3 \\
54 & 13 & 156 & -22 \\
-13 & -3 & -22 & 4
\end{array}\right] \quad \mathrm{j}=1,2, \ldots, \mathrm{n}
$$

Applying the boundary conditions in the above mentioned Eqs. (15 and 16);

$$
\mathrm{Y}_{0}=\mathrm{Y}_{10}=0
$$

Striking out the first row and column from $\mathrm{K}_{1}$ and $\mathrm{M}_{1}$ and the third row and column from $\mathrm{K}_{10}$ and $\mathrm{M}_{10}$ the modified element stiffness matrices for each element is as under;

$$
K_{1}=\frac{E I 10^{3}}{L^{3}}\left[\begin{array}{ccc}
4 & -6 & 2 \\
-6 & 12 & -6 \\
2 & -6 & 4
\end{array}\right], K_{2-9}=\frac{E I 10^{3}}{L^{3}}\left[\begin{array}{cccc}
12 & 6 & -12 & 6 \\
6 & 4 & -6 & 2 \\
-12 & -6 & 12 & -6 \\
6 & 2 & -6 & 4
\end{array}\right], K_{10}=\frac{E I 10^{3}}{L^{3}}\left[\begin{array}{ccc}
12 & 6 & 6 \\
6 & 4 & 2 \\
6 & 2 & 4
\end{array}\right]
$$

The corresponding element mass matrices can be obtained as;

$$
M_{1}=\frac{m L}{4200}\left[\begin{array}{ccc}
4 & 13 & -3 \\
13 & 156 & -22 \\
-3 & -22 & 4
\end{array}\right], M_{2-9}=\frac{m L}{4200}\left[\begin{array}{cccc}
156 & 22 & 54 & -13 \\
22 & 4 & 13 & -3 \\
54 & 13 & 156 & -22 \\
-13 & -3 & -22 & 4
\end{array}\right], \quad M_{10}=\frac{m L}{4200}\left[\begin{array}{ccc}
156 & 22 & -13 \\
22 & 4 & -3 \\
-13 & -3 & 4
\end{array}\right]
$$

\section{Results And Discussion}

Putting the global matrices in the Eq. (13) to solve the eigenvalue problem as a function of concentration of nanoparticles, the approximate eigenvalues and associated eigenvectors for first twenty modes of each sample can be obtained. The behavior of nanocomposite under static straining conditions as well as free vibration considering simply supported nanocomposite beam subjected to initial excitation was studied since it follows a harmonic motion. The storage modulus and density of rubber nanocomposite were found to be a function of volume fraction of filler nanoparticles; hence a much calculation was required to solve the Eq. (13) corresponding to each volume concentration of nanofillers. The mathematical model was simulated in

\begin{tabular}{|c|c|c|c|c|c|c|c|}
\hline \multirow{2}{*}{$\begin{array}{l}\text { Sr. } \\
\text { No. }\end{array}$} & \multirow{2}{*}{$\begin{array}{c}\text { Vol. Fraction of } \\
\text { Nanoparticles } \\
\text { (vf-\%) }\end{array}$} & \multirow[b]{2}{*}{ Method } & \multicolumn{4}{|c|}{ Natural Frequencies $(\mathrm{Hz})$ for First Five Modes } & \multirow[b]{2}{*}{ Mode5 } \\
\hline & & & Mode1 & Mode2 & Mode3 & Mode4 & \\
\hline \multirow{2}{*}{1} & \multirow{2}{*}{0} & Analytical & 4.2625 & 17.05 & 38.363 & 68.2 & 106.56 \\
\hline & & FEM & 4.2624 & 17.051 & 38.382 & 68.311 & 106.98 \\
\hline \multirow{2}{*}{2} & \multirow{2}{*}{5} & Analytical & 4.5742 & 18.297 & 41.168 & 73.187 & 114.36 \\
\hline & & FEM & 4.5741 & 18.298 & 41.189 & 73.307 & 114.8 \\
\hline \multirow{2}{*}{3} & \multirow{2}{*}{8} & Analytical & 4.8724 & 19.49 & 43.852 & 77.958 & 121.81 \\
\hline & & FEM & 4.8723 & 19.491 & 43.874 & 78.086 & 122.29 \\
\hline \multirow{2}{*}{4} & \multirow{2}{*}{10} & Analytical & 5.1282 & 20.513 & 46.154 & 82.051 & 128.2 \\
\hline & & FEM & 5.1281 & 20.514 & 46.177 & 82.185 & 128.71 \\
\hline \multirow{2}{*}{5} & \multirow{2}{*}{12} & Analytical & 5.404 & 21.616 & 48.636 & 86.464 & 135.1 \\
\hline & & FEM & 5.4039 & 21.618 & 48.661 & 86.606 & 135.63 \\
\hline \multirow{2}{*}{6} & \multirow{2}{*}{15} & Analytical & 5.8519 & 23.408 & 52.667 & 93.631 & 146.3 \\
\hline & & FEM & 5.8518 & 23.41 & 52.694 & 93.783 & 146.87 \\
\hline
\end{tabular}
MATLAB to extract the eigenvalues and hence the respective natural frequencies for each sample and the result were tabulated and compared with their counterpart by analytical method in Table 3. In calculating the natural frequencies theoretical values of density and modulus as mentioned in the above Table 2 is used.

Table3. Natural Frequencies as a Function of Nanoparticles Concentration 
Natural frequencies by analytical method, Eq. (8) and that by FEM, Eq. (13) for first twenty modes were plotted in the Fig. 5. The same variation between analytical results and FEM results of natural frequencies is observed for all samples; hence the characteristic for one sample is illustrated. It was clearly indicated that for first five modes the result was almost same by both the techniques and for higher modes a considerable deviation was recorded. As concentration of filler ingredients increases the natural frequency of fundamental mode and higher modes also increases. The normalized eigenvectors corresponding to first five modes were plotted in the Fig. 6.

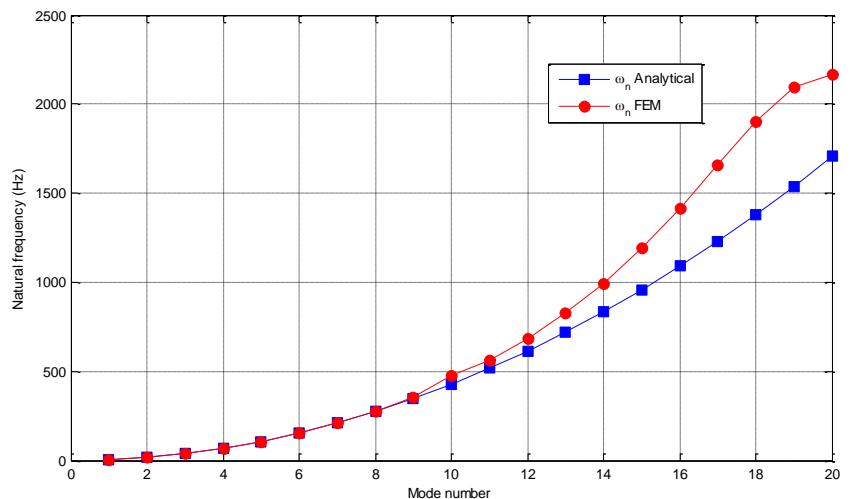

Fig. 5. Comparison of natural frequencies by analytical method and FEM

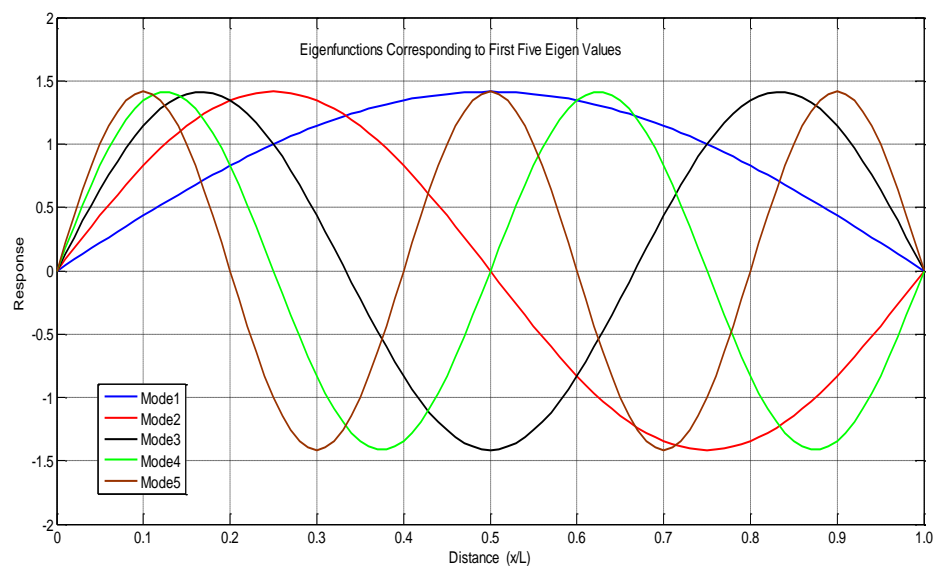

Fig. 6. Normalized mode shapes corresponding to first five modes

The response to initial excitation of nanocomposite system with different volume fractions of aluminazirconia nanoparticles given by the Eq. (11) were plotted overlapped in the Fig. 7 corresponding to the frequency of fundamental mode of each sample. Similarly the performance can be analysed corresponding to the vibration amplitudes of higher modes. The amplitude decay after 10 and 11 seconds can be observed in the zoomed views illustrated in the frames 1 and 2 respectively of Fig. 7. The immediacy of the response can be noted from the phase-lag reduction with increasing nanofiller concentration. The response after 20 seconds was zoomed in the frame 3 of Fig. 7 which indicates the amplitude of 15\% concentrated sample almost dies down to zero, whereas the virgin natural rubber still indicates oscillation. The overall amplitude decay time for fundamental mode of virgin sample was more than 20 seconds and that for $15 \%$ concentrated sample was about 15.5 seconds, which is approximately $25 \%$ less. 


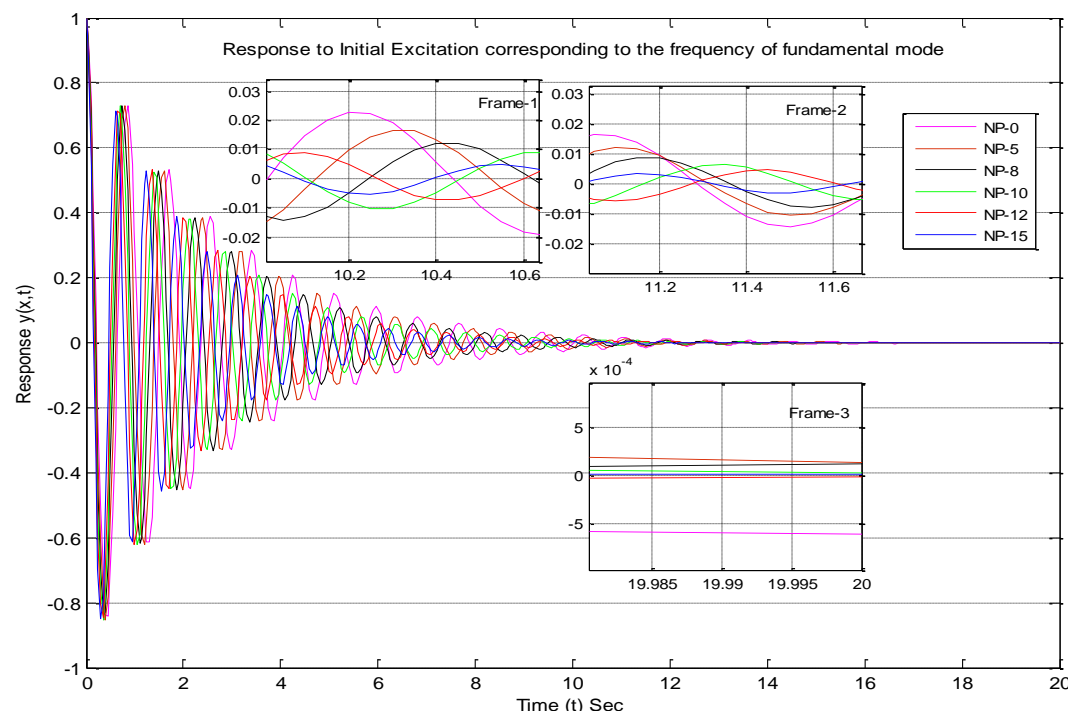

Fig. 7. Response to initial excitation; Superimposed amplitude for all samples corresponding to the frequency of fundamental mode Amplitude decay after $10 \mathrm{sec}, 11 \mathrm{sec}$ and $20 \mathrm{sec}$ is illustrated in the frames 1, 2 and 3 respectively

\section{Micromechanics}

Nanoparticles can accommodate in the voids at favorable geometric orientations of the molecular structure of rubber. The ability of the rubber molecular chains to form nano or microscale-diameter spheres around individual nanoparticles or small cluster of nanoparticles favors the formation of a strong bond with the rubber matrix [24]. Size of reinforced nanoparticles may vary from 1 to $100 \mathrm{~nm}$ in diameter and/or length.

The time to decay the amplitude at the midpoint of the nanocomposite specimen decreases with increase in the concentration of filler nanoparticles which is caused by strong filler-polymer interactions due to nanoparticles-matrix tethering, uniform nanoscale dispersion, and larger surface area of nanoparticles exposed to polymer chains [25]. When an elastomer is reinforced with nano size fillers the entanglement chains causes the stress concentration at microscopic orientations of nanocomposite structure due to clustering of nanoparticles, so the storage modulus rises [26] increasing the overall stiffness of the material. The strong intramolecular attractive forces via hydrogen bonding between rubber matrix and the hydroxyl groups present at the surface of $\mathrm{Al}_{2} \mathrm{O}_{3}-\mathrm{ZrO}_{2}$ are also responsible for the stress-stiffening effect. This phenomenon is also validated by the viscoelastic effect under the increasing rate of strain.

\section{Filler Concentration Sensitivity}

The storage modulus of nanocomposite NR is found to be a function of nanofiller concentration (Fig. 8). The three main medium of energy absorption in the nanocomposite were predicted to be rubber matrix, matrixfiller interaction and the interaction between filler nanoparticles themselves. The loss of energy due to stick-slip motion between filler-matrix interaction and between nanoparticles themselves was the major cause of the improvement in storage modulus. The inherent stiffness of the material has a great effect on amplitude decay and the vibrational energy consumption of the system for the same level of external damping provided.

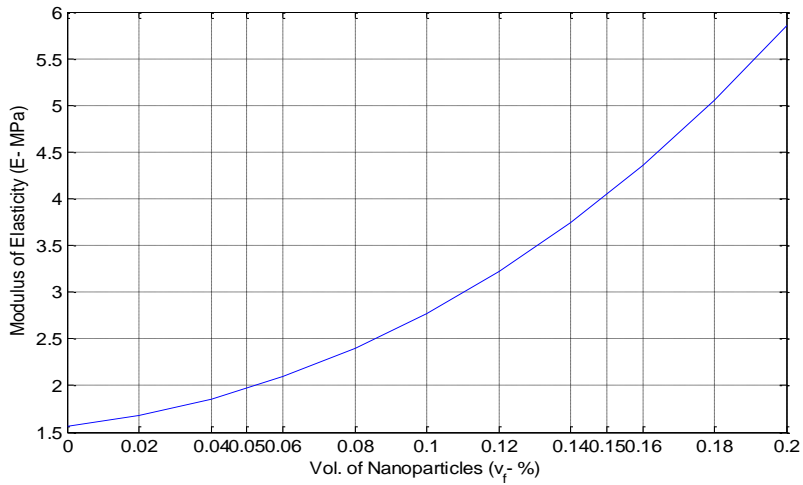

Fig. 8. Sensitivity to nanofiller concentration for storage modulus: The concentration of nanofiller increases the energy consumption micromechanisms, which causes excellent improvement in storage modulus 


\section{Conclusion}

To explore the influence of different concentrations of $\mathrm{Al}_{2} \mathrm{O}_{3}-\mathrm{ZrO}_{2}$ nanoparticles reinforcement on the static and dynamic behavior of the natural rubber and to establish a reasonable ground for designing nanocomposite vibration dampers, the dependence of response on the initial excitation was studied. The results describe the improvement in the viscoelastic properties of natural rubber nanocomposite as a function of its nanostructure. The vibration amplitude decay time is found to be reduced by $25 \%$ due to enhancement in overall stiffness and storage modulus is improved by $260 \%$ approximately. The polyisoprene- $\mathrm{Al}_{2} \mathrm{O}_{3}-\mathrm{ZrO}_{2}$ hybrid nanocomposite samples were successfully prepared by two roll mill process. The structure property relationship in nanocomposites can provide a useful means to determine the extent to which nanofillers has been exfoliated and dispersed in polymer matrix.

\section{Acknowledgement}

The authors gratefully acknowledge the instrumentation facility provided by SAIF, IIT Bombay, Mumbai for TEM and UICT, NMU, Jalgaon for SEM.

There is no specific grant from any funding agency in the public, commercial, or not-for-profit sectors.

\section{References}

[1] R.J. Schaefer, C.M. Harris and A.G. Piersol (Ed.), Harris' shock and vibration handbook 5 (New York: McGraw-Hill Handbooks, 2002).

[2] M. Hernández, M.M. Bernal, R. Verdejo, T.A. Ezquerra and M.A. López-Manchado, Overall performance of natural rubber /graphene nanocomposites, Composites Science and Technology, 73, 2012, 40-46.

[3] Q.X. Jia, Y.P. Wu, Y.Q. Wang, M. Lu and L.Q. Zhang, Enhanced interfacial interaction of rubber/ clay nanocomposites by a novel two-step method, Composites Science and Technology, 68(3-4), 2008, 1050-1056.

[4] R. Schuster, Filler reinforcement. In: Educational Symposia, IRC ’03, Nürnberg, Germany, 2003.

[5] S.H. Avner, Introduction to physical metallurgy (New Delhi: Tata McGraw Hill Edition, 1997).

[6] S. Najidha, N.S. Saxena, R. Sreeja, C.H. Unnithan and P. Predeep, Optical and electrical characterization of SbCl5 doped cis-1,4polyisoprene, Materials Letters, 59, 2005, 3431-3436.

[7] T.H. Lowery and K.S. Richardson, Solvent, mechanism and theory in organic chemistry, (New York: Harper Collins, 1998).

[8] M. Parthasarathy and D.J. Klingenberg, Electrorheology: Mechanisms and models, Materials Science and Engineering: R: Reports, 17, 1996, 57-103.

[9] D.F. Rob and M. Taalman, Isoprene: Background and issues. Toxicology, 113, 1996, 242-246.

[10] E. Dorre and H. Hubner, Alumina (New York: Springer-Verlag, 1984).

[11] T. Mirfakhrai, J.D.W. Madden and R.H. Baughman, Polymer artificial muscles, Materials Today, 10, 2007, 30-38.

[12] E.A. Moschou, M.J. Madou, L.G. Bachas and S. Daunert, Voltage-switchable artificial muscles actuating at near neutral pH, Sensors and Actuators B, 115, 2006, 379-383.

[13] T. Shiga, Deformation and viscoelastic behavior of polymer gels in electric fields. In Neutron Spin Echo Spectroscopy Viscoelasticity Rheology, Springer Berlin Heidelberg, 1997, 131-163.

[14] C.W. Wu and H. Conrad, Electrical properties of electrorheological particle clusters, Materials Science and Engineering A, 255, 1998, 66-69.

[15] T. Mitsumata, K. Sugitani and K. Koyama, Electrorheological response of swollen silicone gels containing barium titanate, Polymer, 45, 2004, 3811-3817.

[16] Y.D. Kim and D.J. Klingenberg, Two roles of nonionic surfactants on the electrorheological response, Journal of Colloid and Interface Science, 183, 1996, 568-578.

[17] Y.D. Kim and I.C. Song, Electrorheological and dielectric properties of polypyrrole dispersions, Journal of Materials Science, 37, 2002, 5051-5055.

[18] M. Liu and M.S. Hoo Fatt, A constitutive equation for filled rubber under cyclic loading, International Journal of Non-Linear Mechanics, 46, 2011, 446-456.

[19] J.Z. Arkadiusz, M.P. Cartmell and W.M. Ostachowicz, A sensitivity analysis of the dynamic performance of a composite plate with shape memory alloy wires, Composite Structures, 60, 2003, 145-157.

[20] D. Sarkar, S. Adak and N.K. Mitra, Preparation and characterization of an $\mathrm{Al}_{2} \mathrm{O}_{3}-\mathrm{ZrO}_{2}$ nanocomposite, Part I: Powder synthesis and transformation behavior during fracture, Composites A: Applied Science and Manufacturing, 38(1), 2007, $124-131$.

[21] F.L. Matthews, Revised primer on composite materials: Analysis (JC Halpin Technomic Publishing Company, Inc., 1984).

[22] L. Meirovitch, Fundamentals of Vibrations (Singapore: McGraw-Hill International edition, 2001).

[23] P.F. Pai and A.H. Nayfeh, Three-dimensional Nonlinear Vibrations of Composite Beams-I. Equations of Motion, Nonlinear Dynamics, 1, 1990 477-502.

[24] A.H. Barber, E. Wiesel and H.D. Wagner, Crack deflection at a transcrystalline junction, Composite Science and Technology, 62, 2002, 1957-1964.

[25] M. Berta, C. Lindsay, G. Pans and G. Camino, Effect of chemical structure on combustion and thermal behaviour of polyurethane elastomer layered silicate nanocomposites, Polymer Degradation and Stability, 91, 2006, 1179-1191.

[26] A.K. Barick and D.K. Tripathy, Effect of organically modified layered silicate nanoclay on the dynamic viscoelastic properties of thermoplastic polyurethane nanocomposites, Applied Clay Science, 52, 2011, 312-321. 